\title{
Concerning the role of perceptual factors in the serial position effect*
}

\author{
GEOFFREY UNDERWOOD \\ University of Nottingham, Nottingham NG7 2RD, England
}

\begin{abstract}
The experiment described here addresses itself to the serial position effect of recall, and in particular to the cause of primacy. Two of the three theories discussed here (selective rehearsal and proactive interference) postulate competition between items during the storage interval as a cause of primacy, acquisition being assumed to be equal for all items. The third theory (differential perceptual processing) places the locus of the effect prior to the storage stage, and does not hold interactions between items to be essential to the effect after encoding. The experiment used this distinction between the two classes of theories. storage of the whole presentation (and hence interactions during storage) not being required. Only one word from each list was recalled, and this item was indicated to $S$ on presentation, thereby eliminating the necessity to attempt to encode all items. The storage-interaction theories predict no primacy for the recall of individual items in this experiment, but the initial member of the list was recalled more of ten than the third member. This example of primacy lends support to the argument that the trace strengths of items are not always equal immediately after presentation.
\end{abstract}

Serial position effects in recall have been demonstrated using a number of techniques--by ordered, anticipated, and free recall and by the various probe recall and recognition methods-and all of these techniques can depend upon a single serial presentation of a list for the effect to be observed. It has been argued that the recall advantage gained by the initial members of a sequential presentation of items (the primacy effect) is a function of selective rehearsal of early items during and after presentation of the list, or a function of proactive interference from early items encouraging the forgetting of later items, or a function of perceptual processing differences within the sequence during the acquisition stage.

One variant of the selective rehearsal hypothesis maintains that more information about early items is stored because the rehearsal strategies employed direct the S's attention to these items (Waugh \& Norman, 1965; Crowder, 1969). The other variant considers that early items enter a relatively empty rehearsal buffer and are not displaced as quickly as are central or late members of the list. More information about early items in the presentation is therefore transferred to the long-term memory store and their recall probability increased (Atkinson \& Shiffrin, 1968; Bruce \& Papay, 1970).

An alternative explanation of the increased recall of early items relies upon the construct of proactive interference (PI). This explanation can be developed from the observation of a decrement in recall as a consequence of the presentation of previous items

*This work was supported by a Research Studentship from the U.K. Science Research Council and is based upon a thesis submitted to the University of Sheffield. I am grateful to Professor Harry Kay for advice and encouragement and to Professors Judith Reitman and Doris Aaronson for their comments on a previous draft of this paper.
(Keppel \& Underwood, 1962; Murdock, 1964). The decrement, said to be due to PI from earlier items causing forgetting or response confusion for new items. can be eliminated by presenting a different class of material as the new items (Wickens, Born. \& Allen, 1963). Primacy may then be due to PI from the early items in the list affecting the recall of succeeding items (Glanzer \& Cunitz, 1966). Early items would not be vulnerable to PI (except from words in other lists), and so recall would not be depressed.

It has also been argued that the recall advantage gained by the initially presented members of a sequence is at least partly a function of intraserial differences in the perceptual processing of items (Aaronson, 1968; Aaronson, Markowitz, \& Shapiro, 1971; Underwood, 1972). The perceptual processing theory considers the intervals between presentation of items and their semantic decoding, and the degree of analysis of items, to be critical variables in the encoding of serial presentations. This theory will now be described in detail.

The work of Efron (1970). Massaro (1970a), Treisman and Rostron (1972), and others indicates the existence of sensory storage of auditory information prior to identification, with similar storage functions to the visual memory described by Sperling $(1960,1967)$. The function of such preperceptual storage would be to maintain the sensory representation of the stimulus beyond the duration of presentation. Aaronson (1967) points out that perceptual analysis can continue after termination of the stimulus presentation, and with short presentation durations, or complex material, such postpresentation analyses of echoic or iconic images may be essential for correct perceptual identification. Preperceptual images of auditory and visual stimuli are subject to rapid decay, estimates of the period beyond which information is irretrievable varying between a few $100 \mathrm{msec}$ and $2 \mathrm{sec}$ (depending largely upon the types of 
infurmation presented). So. an item can be stored as a sensory representation and analyzed some time after presentation. but if the identification delay exceeds a certain critical value the representation will have decayed and not be available for analysis. Similarly. an item which has partially decayed may be imperfectly analyzed and hence an imperfect representation may be stored in postperceptual memory. For example, if the item "P" is spoken to a $\mathrm{S}$ who does not perform an immediate analysis because he is engaged in another task. then only gross acoustical features may be available by the time he gets around to analyzing the word. What is stored in this case may not be the item presented, but any of the numerous other items with the same acoustical features. With both the active and postponed identification strategies described by Aaronson (1968), the items presented later in the sequence are subject to greater identification delays than are items presented early. and so would be in a more decayed state when analyzed. Thus, late items in the list are argued to have greater identification delays than do early items. and so less perfect representations are available for recall.

The second variable relevant to the perceptual processing theory of primacy is that of the time available for analysis. Massaro (1970b) asserts that memory strength increases exponentially with an increase in the perceptual processing of the item, and so recall differences may be accountable to differences in the time available for initial inspection. With the presentation of a list, serial position curves may be due to longer processing of early items than of succeeding items. The initial members of the list may have such a processing advantage due to strategical factors. The $\mathrm{S}$ may choose to attend selectively to the beginning of the list because of the change in stimulus presentation: the first item follows a period of nonstimulation, but the second item is preceded by a similar stimulus (Kintsch, 1970). Alternatively, the early items may demand attention due to their distinctiveness (Murdock, 1960) in comparison with preceding stimulation. Such differences in the strategical distribution of attention within a list may correspond to the selection of "anchor points" in the serial learning situation, as described by Feigenbaum and Simon (1962).

The perceptual processing theory of primacy states that initial items may be more efficiently analyzed than centrally presented items, resulting in stronger memory traces and hence higher recall probabilities of early than of central and late items. Selective rehearsal and proactive interference theories, however, consider the locus of the primacy effect to be after initial encoding: they assume that all items are encoded equally efficiently and that primacy is a function of selective processing or differential forgetting during storage. To eliminate the possibility of interactions between items during the storage period. and hence to test these two categories of theories, the method used in the experiment described here required Ss to retain only onc item from each list and to respond with this item at the end of the presentation. If perceptual processing is a factor which contributes to the primacy effect, and if sequential presentations result in less efficient processing of central items than of initial items, then a primacy effect should be observed with this technique, as retention is still a function of the efficiency of perception. Rehearsal theory should not predict such a primacy effect. because there is no reason why Ss should selectively rehearse the first item of the series when indicated as the recall item rather than rehearse a central item when it is required. As only one item from the list had to be retained from each list, storage interference effects should also be minimal, but incidental retention of noncritical items may lead to a certain amount of PI influencing the recall of central items more than that of initial items. However, in an experiment reported by Bruce and Papay (1970), presentation of items subsequently cued as "to be forgotten" had little effect on primacy, suggesting that intraserial PI effects from incidental material are minimal.

The item for recall was designated at the instant of presentation by the occurrence of a tone in the opposite ear to that of presentation of the set of verbal items. This method differs from the usual probe recall method of testing for partial recall of the list. With the probe recall paradigm of Waugh and Norman (1965), output interference effects are eliminated, but acquisition, storage, and retrieval processes still remain as possible causes of the (reduced) primacy effect. With the probe technique, Ss are required, ideally, to store the whole of the presentation until the probe itself is presented. The present technique of indicating the critical item to the $S$ at the time of presentation eliminates the possibility of interactions between items during the storage interval: retention is a function of perceptual analysis and encoding and of subsequent forgetting of a single item.

To prevent retention of the cued item by selective rehearsal. Ss were required to shadow (overtly rehearse) the whole list. Underwood and Moray (1971) criticized the employment of the shadowing response in experiments seeking an absolute measure of the detectability of target items, but in the present situation the attention-directing and capacity-consuming features of the response were of advantage. Simply asking Ss not to rehearse selectively, or telling them to think only of the last item presented would not control their strategies: these instructions, used by Waugh and Norman (1965), can only be effective when $S$ does not know in advance which item will be critical. Levy and Jowaisas (1971) found recall decrements when items semantically similar to the target item were shadowed during the interval between presentation and recall of the critical item. Kroll. Parks. Parkinson. Bieber. and Johnson (1970) similarly found that the failure of recall of a single spoken item was a function of the amount of retroactive interference $(\mathrm{R} I)$ from the interpolated shadowing task. Semantic interference alone should 
depend only on the number of interpolated items. according to this finding, with no increase in the probability of recall of initially presented items. Trace decay theory would also predict a recall probability function which varies inversely with the time between presentation and recall, provided that $S$ s were not rehearsing the critical item. Again, no primacy should be expected if this is the only factor operating.

\section{METHOD}

\section{Apparatus and Stimulus Materials}

Forty lists. each composed of four randomly selected digits ( 1 through 10 , excluding 7) followed by 10 randomly selected letter names (excluding W). were recorded on Channel 1 of a Sonv TC-500a stereo tape recorder, with a presentation rate of $1.5 \mathrm{items} / \mathrm{sec}$ in each list. Repeats of items within each list were eliminated. The digits and letters were read, by a male speaker, as a continuous string in each list, with equal pauses between each of the 14 items. The purpose of the digits was to ensure that shadowing had commenced at the time of presentation of the first letters. Without the digits at the beginning of the lists, the first few letters would have been. perceived more clearly than the remaining letters because of the absence of stimulus masking from the shadowing voice. The letter for recall was designated by the recording of a $100-\mathrm{msec}$ tone (approximately $900 \mathrm{~Hz}$ ) on Channel 2 of the tape recorder at the onset of the appropriate item. The tone accompanied a letter in each of the 10 serial positions four times, with random selection of the critical position within this constraint. A relatively slow presentation rate was used because perfect shadowing performances, collected on a second tape recorder, were demanded from Ss. In practice, all lists were scored, regardless of whether or not perfect shadowing had been observed. Several more lists were recorded to give practice at the shadowing task, and three lists similar to the experimental lists were prepared for pratice at the combined tasks. Presentation of stimuli was over Eagle SE-1 stereo earphones.

\section{Subjects}

Twelve females and eight males, between the ages of 18 and 25 years, were drawn from a volunteer $S$ pool composed of undergraduate and research students from the University of Sheffield. All Ss claimed to be right-handed.

\section{Procedure}

Half of the Ss were presented with verbal material to their right ears (and therefore tones to their left ears), and half were presented with the lists of digits and letters to their left ears. Lists of letters were first presented for shadowing practice until a criterion of three successive errorless lists was achieved. The three practice trials of shadowing and remembering the critical item were then given. To minimize selective rehearsal of the memorized letter, emphasis on perfect shadowing was made in the instructions: $S$ s were told that only those trials in which errorless shadowing was obtained would be scored on the memory task. Ss were instructed that they should remember the letter that occurred at the same time as the tone and to report verbally that letter only after completing the shadowing task. The 40 experimental trials were presented with only a short break between trials.

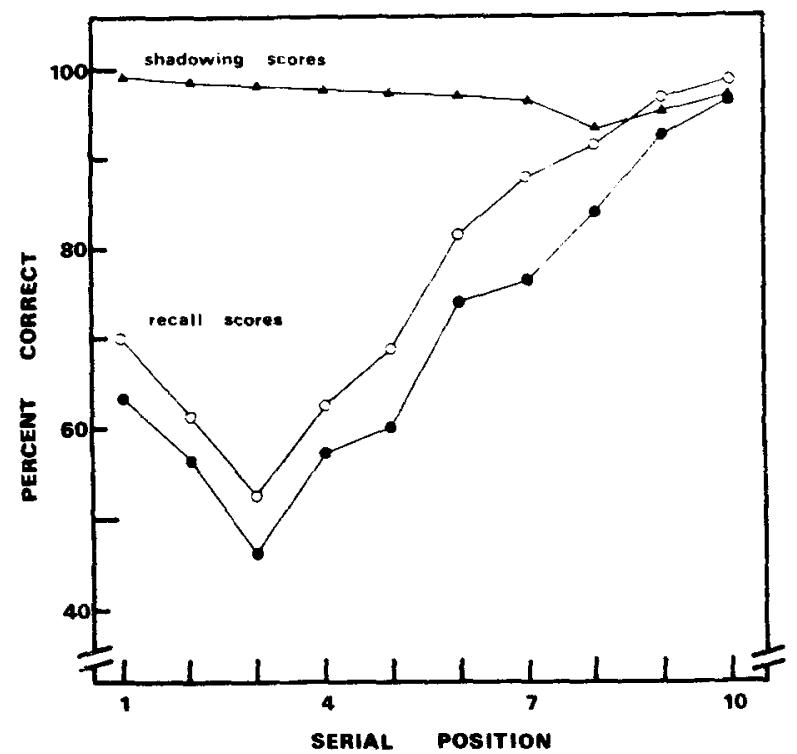

Fig. 1. Shadowing and critical-item recall scores. correct recall of critical item ; $0-0$ recall of critical item + adjacent recall errors.

\section{RESULTS}

\section{Position Effects}

As illustrated in Fig. 1, recall of items presented in Serial Position 1 (63.75\%) was greater than recall of items in Serial Position $3(46.25 \%)$, thus confirming the prediction derived from the perceptual processing theory of primacy $(\mathrm{t}=2.77, \mathrm{df}=19, \mathrm{p}<.01$, one-tailed $)$.

Recall errors were classified into two general types: omissions and guesses, and recall of items adjacent to the critical item. Ss who offered an adjacent item as the recall response appeared unaware that they were in error and gave this item immediately and confidently. The other error category was characterized by the $\mathrm{S}$ either failing to recall at all or hesitating for a few seconds and then offering an incorrect letter, which was occasionally a letter which had been presented towards the end of the list. These errors may have been caused by interference or decay effects, whereas adjacent recall errors suggest a failure of perceptual integration. This view is supported by the absence of any systematic interaction between the number of these errors over time. The relationships between adjacent recall errors and correct recall are illustrated in Fig. 1. Ladefoged and Broadbent (1960) reported an experiment in which clicks were superimposed over lists of digits, one click during each list of 10 digits, and the S's task was to remember the digit occurring at the same time as the click. The tendency was for Ss to report the click as occurring earlier in the list than was the case, and the authors discussed the result in terms of the theory of prior entry, which states that we perceive events which we are 
expecting more quickly than we do other events. Hence, the S would be predisposed towards, or have a "set" towards. hearing the click and so may associate this event with an item earlier than the item which is simultaneously presented but which is still being processed. In the present experiment, however, the tendency to offer earlier items was not so pronounced in comparison with the offer of later items $(t=1.25, \mathrm{df}=$ 19 , n.s.). The effect may have been confounded by the shadowing activity. The shadowing may. for instance, have reduced the subjective set for the tone by directing the S's attention towards the verbal material.

\section{Ear Differences}

From the groups of $S$ s listening to the stimuli with different ears came a curious, though nonsignificant, trend. The work of Kimura (1961, 1964), Bryden (1967). Darwin (1971), and others indicates a right-ear advantage (REA) for the perception of verbal material and a left-ear advantage (LEA) for the perception of nonverbal material such as clicks, tones, and melodies. In the present experiment, in which verbal and nonverbal material were presented simultaneously to opposite ears, it might be expected, therefore, that Ss who were presented with verbal material to their right ears and tones to their left ears would perform better than Ss in the reverse condition, who would not be given the independent-ear advantages for perception defined by the previous asymmetry work. This was not the case. When letters were presented to Ss' left ears, critical items were recalled on $77.5 \%$ of all occasions, whereas when the critical item was presented to the right ear, the recall rate was $66.25 \%(\mathrm{~F}=3.18, \mathrm{df}=1,18, \mathrm{n.s}$.$) .$

\section{DISCUSSION}

\section{Position Effects}

The experiment reported here did find a deviation from the exponential decay or cumulative interference function which might be expected if each item had an equal initial representation in memory. Whereas the probability of recall decreased as the time and number of items between presentations and retrieval increased up to a point, the items presented in Serial Position 1 were recalled more often than those presented in Serial Position 3, despite the extra decay and/or interference to which the earlier items were subject. This result creates a difficulty for accounts of primacy based on rehearsal and on proactive interference, for both of these theories postulate interactions between the items of the list during the storage processes to be the cause of the effect. Such interactions were not present in this experiment. as only one item per trial was stored for subsequent retrieval. This assertion is dependent. however. upon two basic assumptions: that the shadowing task effectively prevents the $S$ from attending to material other than that being shadowed, and that PI from items not designated as critical, but which may be incidentally retained, is minimal. Time/item decay and RI predict the decrease in recall with earlier presentation position, but similarly cannot account for the increase in recall over the initial serial positions. If items receiving attention gain differential processing according to their input serial positions, however, with early items being processed more efficiently because they are subject to shorter delays of perceptual analysis than are later iterns, then the resultant stronger traces of initial items might override any decay/interference influences more effectively than those items whose traces are weak through insufficient processing.

It is further possible that the primacy effect would have been magnified in the absence of the four digits, which might have reduced the perceptual distinctiveness of the initial letters. These digits were redundant to the memory task, but were necessary to avoid differential masking by the shadowing voice within the series of letters. The primacy here, therefore, reflects the semantic distinctiveness of the initial letters: they are from a different semantic category to their preceding items, and this distinctiveness may have enhanced their processing and encoding when indicated as critical. However, while the listener was analyzing the final digit, a refractory period effect may have impaired the perception of the following item, the first letter, and hence reduced the primacy.

\section{Ear Differences}

Apart from the difficulty of explaining a result which is so inconsistent with the literature-that of a LEA for verbal material-two further problems are present. First, independent groups of Ss yielded the data for the ears comparison, whereas within-S comparisons are preferable when investigating slight differences which should be present for each individual but which have considerable between-S variation. Second, the Ss were required to shadow the material presented. Springer (1971) found that the REA for verbal material is a perceptual phenomenon and not simply a function of the asymmetry of verbal output, but this does not exclude the possibility of the continuous verbal transcription of a message influencing the lateralization of the processing stages.

Rosenzweig (1951) and Kimura (1961) consider the asymmetry of auditory perception to be due to the superiority of contralateral pathways over ipsilateral pathways. The left hemisphere is generally dominant in the perception of verbal material and hence such material is perceived better when presented to the right ear than when presented to the left. The "split brain" experiments (Gazzaniga \& Sperry, 1967: Sperry. 1968) indicate that both hemispheres are capable of manipulating verbal material, but that only the Jominant hemisphere is able to control the speech 
mechanisms. Shadowed material would then have to be processed by the left hemisphere, at least at the output stage, although it is possible that the two types of material may have been processed by either of the hemispheres in the present experiment. Treisman and Geffen (1968) also consider the possibility of speech messages being monitored by the right hemisphere under certain circumstances. The necessity for the integration of the two sources of information for the $S$ to tag one letter as being critical argues against the total separation of processes, but one highly speculative explanation of the curious effect observed here is that the most efficient way of handling simultaneous verbal and nonverbal information presented to different ears is to avoid multiple interhemispheric transfers by distributing information arriving in each ear along the ipsilateral pathways. This would be of particular benefit in the shadowing situation where verbal material presented to the left ear would be perceived and output without any interhemispheric transfer of information.

\section{REFERENCES}

Aaronson, D. Temporal factors in perception and short-term memory. Psychological Bulletin, 1967, 67, 130-144.

Aaronson, D. Temporal course of perception in an immediate recall task. Journal of Experimental Psychology, 1968, 76, 129-140.

Aaronson, D., Markowitz, N., \& Shapiro, H. Perception and immediate recall of normal and "compressed" auditory sequences. Perception \& Psychophysics, 1971, 9, 338-344.

Atkinson, R. C., \& Shiffrin, R. M. Human memory: A proposed system and its control processes. In K. W. Spence and J. T. Spence (Eds.), The psychology of learning and motrivation: Advances in research and theory. Vol. 2. London: Academic Press, 1968.

Bruce, D., \& Papay, J. P. Primacy effect in single-trial free recall. Journal of Verbal Learning \& Verbal Behavior, 1970, 9, 473-486.

Bryden, M. P. An evaluation of some models of laterality effects in dichotic listening. Acta Oto-Laryngologica, 1967, 63, 595-604.

Crowder, R. G. Behavioral strategies in immediate memory. Journal of Verbal Learning \& Verbal Behavior, 1969, 8, 524-528.

Darwin, C. J. Ear differences in the recall of fricatives and vowels. Quarterly Journal of Experimental Psychology, 1971, $23,46-62$.

Efron, R. Effect of stimulus duration on perceptual onset and offset latencies. Perception \& Psychophysics, 1970, 8, 231-234.

Feigenbaum, E. A., \& Simon, H. A. A theory of the serial position effect. British Journal of Psychology, 1962, 53, 307-320.

Gazzaniga, M. S., \& Sperry, R. W. Language after section of the cerebral commissures. Brain, 1967, 90, 131-148.

Glanzer, M., \& Cunitz, A. R. Two storage mechanisms in free recall. Journal of Verbal Learning \& Verbal Behavior, 1966, 5 , 351-356.
Keppel, G. \& Linderwood. B. J. Proactive inhibition in short-term retention of single jtems. Journal of Verbal Learning \& Verbal Behavior. 1962. 1. 153-161.

Kimura. D. Cerebral dominance and the perception of verbal stimuli. Canadian Journal of Psychology. 1961. 15. 166-171.

Kimura, D. Left-right differences in the perception of melodies Quarterly Journal of Experimental Psychology, 1964. 14. 355-358.

Kintsch. W. Learning. memory, and conceptual processes. New York: Wiley, 1970.

Kroll, N. E. A., Parks. T., Parkinson. S. R., Bieber. S. L.. \& Johnson, A. L. Short-term memory while shadowing: Recall of visually and of aurally presented letters. Journal of Experimental Psychology; 1970. 85, 220-224.

Ladefoged, P., \& Broadbent, D. E. Perception of sequence in auditory events. Quarterly Journal of Experimental Psychology, 1960, 12, 162-170.

Levy, C. M., \& Jowaisas, D. Short-term memory: Storage interference or storage decay? Journal of Experimental Psychology, 1971, 88, 189-195.

Massaro, D. W. Preperceptual auditory images. Journal of Experimental Psychology. 1970a. 85, 411-417.

Massaro, D. W. Perceptual processes and forgetting in memory tasks. Psychological Review, 1970b. 77. 557-567.

Murdock, B. B., Jr. The distinctiveness of stimuli. Psychological Review, 1960, 67. 16-31.

Murdock, B. B.. Jr. Proactive inhibition in short-term memory: Journal of Experimental Psychology' 1964. 68. 184-189.

Rosenzweig, M. R. Representations of the two ears at the auditory cortex. American Journal of Physiology. 1951. 167. $147-158$.

Sperling, G. The information available in brief visual presentations. Psychological Monographs, 1960. 74(11. Whole No. 498).

Sperling, G. Successive approximations to a model for short-term memory. Acta Psychologica, 1967, 27. 285-292.

Sperry, R. W. Hemispheric deconnection and unity in conscious awareness. American Psychologist, 1968, 23. 723-733.

Springer, S. P. Ear asymmetry in a dichotic detection task. Perception \& Psychophysics, 1971, 10, 239-241.

Treisman, A. M., \& Geffen, G. Selective attention and cerebral dominance in perceiving and responding to speech messages. Quarterly Journal of Experimental Psychology, 1968, 20. 139-150.

Treisman, M., \& Rostron. A. B. Brief auditory storage: A modification of Sperling's paradigm applied to audition. Acta Psychologica, 1972, 36, 161-170.

Underwood, G. Response organization in attention control and a perceptual serial position effect. Quarterly Journal of Experimental Psychology, 1972, 24, 340-351.

Underwood, G., \& Moray, N. Shadowing and monitoring for selective attention. Quarterly Journal of Experimental Psychology, 1971, 23, 284-295.

Waugh. N. C., \& Norman, D. A. Primary memory. Psychological Review, 1965, 72, 89-104.

Wickens, D. D., Born, D. G., \& Allen, C. K. Proactive inhibition and item similarity in short-term memory. Journal of Verbal Learning \& Verbal Behavior, 1963, 2, 440-445.

(Received for publication July 17, 1972; revision received December 11,1972 .) 